\title{
Colonic metaproteomic signatures of active bacteria and the host in obesity
}

Citation for published version (APA):

Kolmeder, C. A., Ritari, J., Verdam, F. J., Muth, T., Keskitalo, S., Varjosalo, M., Fuentes, S., Greve, J. W., Buurman, W. A., Reichl, U., Rapp, E., Martens, L., Palva, A., Salonen, A., Rensen, S. S., \& de Vos, W. M. (2015). Colonic metaproteomic signatures of active bacteria and the host in obesity. Proteomics, 15(20), 3544-3552. https://doi.org/10.1002/pmic.201500049

Document status and date:

Published: 01/10/2015

DOI:

10.1002/pmic.201500049

Document Version:

Publisher's PDF, also known as Version of record

Document license:

Taverne

Please check the document version of this publication:

- A submitted manuscript is the version of the article upon submission and before peer-review. There can be important differences between the submitted version and the official published version of record.

People interested in the research are advised to contact the author for the final version of the publication, or visit the DOI to the publisher's website.

- The final author version and the galley proof are versions of the publication after peer review.

- The final published version features the final layout of the paper including the volume, issue and page numbers.

Link to publication

\footnotetext{
General rights rights.

- You may freely distribute the URL identifying the publication in the public portal. please follow below link for the End User Agreement:

www.umlib.nl/taverne-license

Take down policy

If you believe that this document breaches copyright please contact us at:

repository@maastrichtuniversity.nl

providing details and we will investigate your claim.
}

Copyright and moral rights for the publications made accessible in the public portal are retained by the authors and/or other copyright owners and it is a condition of accessing publications that users recognise and abide by the legal requirements associated with these

- Users may download and print one copy of any publication from the public portal for the purpose of private study or research.

- You may not further distribute the material or use it for any profit-making activity or commercial gain

If the publication is distributed under the terms of Article $25 \mathrm{fa}$ of the Dutch Copyright Act, indicated by the "Taverne" license above, 


\title{
Colonic metaproteomic signatures of active bacteria and the host in obesity
}

\author{
Carolin A. Kolmeder ${ }^{1}$, Jarmo Ritari ${ }^{1}$, Froukje J. Verdam ${ }^{2}$, Thilo Muth ${ }^{3}$, Salla Keskitalo ${ }^{4}$, \\ Markku Varjosalo ${ }^{4}$, Susana Fuentes ${ }^{5}$, Jan Willem Greve ${ }^{2}$, Wim A. Buurman ${ }^{2}$, Udo Reichl3,6, \\ Erdmann Rapp ${ }^{3}$, Lennart Martens ${ }^{7,8}$, Airi Palva ${ }^{1}$, Anne Salonen ${ }^{9}$, Sander S. Rensen ${ }^{2}$ \\ and Willem M. de Vos ${ }^{1,5,9}$ \\ ${ }^{1}$ Department of Veterinary Biosciences, University of Helsinki, Helsinki, Finland \\ 2 Department of General Surgery, NUTRIM School of Nutrition and Translational Research in Metabolism, \\ Maastricht University Medical Center, Maastricht, The Netherlands \\ ${ }^{3}$ Max Planck Institute for Dynamics of Complex Technical Systems, Bioprocess Engineering, Magdeburg, Germany \\ ${ }^{4}$ Institute of Biotechnology, University of Helsinki, Helsinki, Finland \\ ${ }^{5}$ Laboratory of Microbiology, Wageningen University, Wageningen, The Netherlands \\ ${ }^{6}$ Chair of Bioprocess Engineering, Otto-von-Guericke University, Magdeburg, Germany \\ ${ }^{7}$ Department of Biochemistry, Ghent University, Ghent, Belgium \\ ${ }^{8}$ VIB Medical Biotechnology Center, Department of Medical Protein Research, Ghent, Belgium \\ ${ }^{9}$ Department of Bacteriology and Immunology, Immunobiology Research Program, University of Helsinki, \\ Helsinki, Finland
}

Obesity is associated with the intestinal microbiota in humans but the underlying mechanisms are yet to be fully understood. Our previous phylogenetic study showed that the faecal microbiota profiles of nonobese versus obese and morbidly obese individuals differed. Here, we have extended this analysis with a characterization of the faecal metaproteome, in order to detect differences at a functional level. Proteins were extracted from crude faecal samples of 29 subjects, separated by 1D gel electrophoresis and characterized using RP LC-MS/MS. The peptide data were analyzed in database searches with two complementary algorithms, OMSSA and X!Tandem, to increase the number of identifications. Evolutionary genealogy of genes: nonsupervised orthologous groups (EggNOG) database searches resulted in the functional annotation of over $90 \%$ of the identified microbial and human proteins. Based on both bacterial and human proteins, a clear clustering of obese and nonobese samples was obtained that exceeded the phylogenetic separation in dimension. Moreover, integration of the metaproteomics and phylogenetic datasets revealed notably that the phylum Bacteroidetes was metabolically more active in the obese than nonobese subjects. Finally, significant correlations between clinical measurements and bacterial gene functions were identified. This study emphasizes the importance of integrating data of the host and microbiota to understand their interactions.

\section{Keywords:}

Composition / Intestinal microbiota / Metaproteomics / Microbiology / Obesity

Additional supporting information may be found in the online version of this article at the publisher's web-site

\footnotetext{
Correspondence: Dr. Carolin A. Kolmeder, Department of Veterinary Biosciences, University of Helsinki, PO Box 66, 00014 Helsinki, Finland

E-mail: carolin.kolmeder@helsinki.fi

Fax: $+358-2491-57033$
}

Received: February 2, 2015

Revised: July 3, 2015

Accepted: July 24, 2015

\begin{abstract}
Abbreviations: AP, alkaline phosphatase; bagged RDA, redundancy analysis using bootstrap aggregation; BMI, body mass index; EggNOG, evolutionary genealogy of genes: nonsupervised orthologous groups; IBD, inflammatory bowel disease; LCA, lowest common ancestor; PCoA, principal coordinate analysis; SCFA, short chain fatty acids
\end{abstract}

Colour Online: See the article online to view Figs. 1-4 in colour 


\section{Significance of the study}

Intestinal microbiota has been associated with obesity, although the exact mechanisms and bacteria involved are largely unresolved. For a better understanding of the metabolic differences between obese and nonobese individuals, functional studies going beyond genome and phylogenetic description of the microbiota are needed due to our limited ability to infer organismal function from 16S rRNA gene sequences. Here, we report the first proteomic study of intestinal content of obese adults and show that both their human and bacterial proteins differ significantly from that of nonobese individuals. While BMI as such did not associate with the abundance of any bacterial or host protein, we identified statistically significant correlations between bacterial gene functions and insulin sensitivity. These data, in parallel to identification of metabolically active members of the microbiota, highlight the potential of functional analyses in defining obesity-related alterations in the gut microbiota as well as in the host.

\section{Introduction}

The epidemic-like spread of obesity over the last half century in all parts of the world and its burden on individuals and society call for efficient treatment strategies. Therefore, mechanistic insight into the multifaceted etiology of the condition is required. To elucidate the causes for the onset of obesity, host (epi-)genome, nutrients, the intestinal microbiota, and the combination of all three factors have to be considered [1]. In particular, the characterization of the intestinal microbiota has gained large research interest in general and especially in the context of obesity over the last decade. Differences in composition and genetic diversity of the intestinal microbiota between lean and obese adults have been identified in several cohorts, although without a clear overlap between these studies, as discussed recently [2]. While the missing consensus may be due to the complexity of the disease and confounding factors, it may also be related to technical aspects, such as limited scope of the used composition-based methods and absence of focus on microbial functionality.

The role of the microbiota in obesity has been suggested to involve various metabolic and signaling systems, including the synthesis of short chain fatty acids (SCFA), the conversion of bile acids, and the production of hormones and other signaling molecules [3,4]. Especially, the role of SCFA has raised considerable discussions as these have been suggested to be positively associated with body mass index (BMI) [5]. In contrast, individuals with higher levels of butyrate producers have been found to be less obese [2]. Most comparisons of the intestinal microbiota between lean and obese adults have been based on 16S rRNA, metagenomics, and other microbiota profiling studies [6]. For a complete understanding of the metabolic differences between obese and nonobese individuals, it is essential to address the biological activity of the intestinal microbiota. Metatranscriptomics has been used to address the functionality of the microbiota using RNA-seq approaches but requires rapid sampling because of the inherent mRNA instability $[7,8]$. Metaproteomic approaches target proteins, which are relatively stable molecules, and have the advantage to simultaneously address the microbiota and host functionality [9-12]. So far, neither mRNA nor proteins of the microbiome of obese adults have been analyzed. However, a recent report described the detailed faecal metaproteomes of an obese and a lean adolescent at the age of 15 years and identified differences in the expressed functions [13]. For a systematic comparison in adults, we studied here the colonic metaproteomes, comprising microbial and human proteins, of a cohort of 29 Dutch adults ranging from lean to morbidly obese and correlated these with the previously determined microbial composition and clinical patient data. We focused on providing a detailed overview of the functional associations between microbial and host proteins in the human intestine in the context of obesity, as well as on the identification of differences in microbial functionality between the obese and nonobese subjects.

\section{Materials and methods}

\subsection{Subjects and samples}

Details of the subject recruitment, sampling, and assessing clinical parameters have been described previously [14]. Altogether, faecal samples from 29 individuals were studied (Supporting Information Table 1). Microarray analysis and metaproteomics were carried out from the very same faecal sample and the phylogenetic measurements of 28 of these faecal samples have been published before [14]. The individuals studied here included nine with normal BMI $\left(18.6-24.6 \mathrm{~kg} / \mathrm{m}^{2}\right)$, four overweight individuals (BMI 25.2$29.6 \mathrm{~kg} / \mathrm{m}^{2}$ ), and 16 obese individuals (BMI $30.5-60.3 \mathrm{~kg} / \mathrm{m}^{2}$ ). To allow a clear-cut differentiation, the subjects were classified into a nonobese (BMI $<30 \mathrm{~kg} / \mathrm{m}^{2}$ ) and an obese group $\left(\mathrm{BMI}>30 \mathrm{~kg} / \mathrm{m}^{2}\right)$. One of the obese individuals was treated for type 2 diabetes and two other were diabetic according to the $\mathrm{HbA}_{1 \mathrm{c}}(\%)$ measurement. While only one subject from the nonobese group reported regular medication other than oral contraceptives, 12 from the obese group reported medication for various reasons, such as for pain relief, depression, or breathing difficulties. There was no obvious difference in the consistency of the faecal material that could have affected DNA or protein extraction efficiency. 
This observational study was approved by the Medical Ethics Committee of the Atrium Medical Center (Heerlen, the Netherlands; registration number NL30502.096.09) and conducted according to the revised version of the Declaration of Helsinki (59. WMA-General Assembly, Seoul, Korea, October 2008). Informed consent in writing was obtained from each subject individually. A subset of the metaproteomes has been used in a study on evaluating the impact of several components of database searching on peptide identification in the case of metaproteomics [15].

\subsection{Protein extraction}

Proteins from frozen faecal material were extracted and fractioned with minor modifications, as described in [11]. In brief, faecal samples were subjected to bead beating in PBS. We used a FastPrep-24 (MP Biomedicals) with a CoolPrep adapter and $0.1 \mathrm{~mm}$ zirconium silicate beads. Samples were kept on ice during the compulsory 5-min cooling steps in-between the five cycles of bead beating. Proteins were separated on a 4 $12 \%$ NuPAGE Bis-Tris gel (Invitrogen), and the 37 and $75 \mathrm{kDa}$ bands of a prestained protein marker (Precision Plus ${ }^{\mathrm{TM}}$ Dual Color, Bio-Rad) were used as reference for cutting the enclosed 37-75 kDa gel region that was subsequently subjected to in-gel digestion, essentially as reported previously $[11,16]$. Peptides were purified with C18 microspin columns (Harvard Apparatus, USA) according to the manufacturer's instructions and redissolved in $30 \mu \mathrm{L}$ of $0.1 \%$ trifluoroacetic acid and $1 \% \mathrm{ACN}$ in HPLC-grade water.

\subsection{LC coupled to MS/MS (LC-MS/MS)}

RP-HPLC online coupled to MS/MS (LC-MS/MS) analysis of the protein digests was carried out on a nano-HPLC system EASY-nLC II (Thermo Fisher Scientific, Germany) connected to an Orbitrap Elite hybrid mass spectrometer (Thermo Fisher Scientific) with a nano-electrospray ion source (Thermo Fisher Scientific). Per LC-MS/MS run, a total of $4 \mu \mathrm{L}$ of sample was injected and analyzed. The tryptic peptides were automatically loaded from the autosampler into a C18-packed precolumn (EASY-Column ${ }^{\mathrm{TM}} 2 \mathrm{~cm} \times 100 \mu \mathrm{m}$, $5 \mu \mathrm{m}, 120 \AA$; Thermo Scientific) at a flow rate of $1 \mu \mathrm{L} / \mathrm{min}$ in $10 \mu \mathrm{L}$ volume of buffer A ( $1 \% \mathrm{ACN}$ and $0.1 \%$ formic acid in HPLC-grade water), transferred onward to a C18-packed analytical column (EASY-Column ${ }^{\mathrm{TM}} 10 \mathrm{~cm} \times 75 \mu \mathrm{m}, 3 \mu \mathrm{m}$, $120 \AA$; Thermo Scientific), and separated starting at $5 \%$ buffer B (98\% ACN and $0.1 \%$ formic acid in HPLC-grade water) for 5 min, followed by a 120 -min linear gradient from 5 to $35 \%$ of buffer B at a flow rate of $300 \mathrm{~nL} / \mathrm{min}$. This gradient was followed by a 5 -min gradient from 35 to $80 \%$ of buffer B, a 1-min gradient from 80 to $100 \%$ of buffer B, and a 9-min column wash with $100 \%$ buffer $\mathrm{B}$, at a constant flow rate of $300 \mathrm{~nL} / \mathrm{min}$.

Full MS scan was acquired in positive ion mode, with a resolution of 60000 , at a normal mass range in the orbitrap analyzer. The method was set to fragment the 20 most intense precursor ions with CID (collision energy 35).

Data acquisition was performed for survey scan (MS) using profile mode carried out in the high-resolution orbitrap mass analyzer and for fragment ion scan (MS/MS) using centroid mode carried out in the linear iontrap mass analyzer. Peak picking was performed by Thermo Proteome Discoverer 1.4.1.14. The resulting spectra were output to MASCOT Generic Format, converted with default settings on the spectrum selector node for the conversion workflow (mass range settings: precursor mass range 350-5000 Da). The MASCOT Generic Format files are accessible ftp://MSV000079035@massive.ucsd.edu and ftp://MSV000079242@massive.ucsd.edu (generic password "a").

\subsection{Peptide spectral matching}

Peptide spectral matching was performed with the two search algorithms X!Tandem (version 2013.02.01) [17] and OMSSA (version 2.1.8) [18] with the following parameters: trypsin was used as a proteolytic enzyme; up to two missed cleavages were allowed; carbamidomethylation of cysteine was chosen as fixed, and oxidation of methionine as variable modification; and the fragment ion tolerance was set to $0.4 \mathrm{Da}$ and the precursor tolerance to $0.03 \mathrm{Da}$. The protein sequence database used was the in-house human intestinal metaproteome database (HIMPdb, 6153068 protein sequences). It was constructed from different sources, such as metagenomes, bacterial genomes, the human genome, as well as plant genomes and therefore represents a wide range of expected proteins in faecal samples (Supporting Information Table 2). The FDR was estimated based on a target-decoy approach with a database that was generated by using the reversed protein sequence database to obtain the decoy sequences. To guarantee consistent FDR estimation across heterogeneous search engines with different scoring techniques, qvality [19] was used for $q$-value (minimum FDR) calculation based on separate target and decoy searches. An FDR filter of $5 \%$ was applied to the results, since such a filter has been used in previous metagenomics studies [11,20]. Moreover, a recent study has justified the use of a 5\% FDR filter, since peptide spectral matching with a database size of several million of protein sequences underestimates false-positive identifications [15]. Spectra with ambiguous peptide identifications were not used for further analysis.

\subsection{Functional and phylogenetic assignment of peptides}

The peptides were functionally assigned according to the target protein sequence they were part of. The target protein sequences were matched to the evolutionary genealogy of genes: nonsupervised orthologous groups (EggNOG) $4.0 \mathrm{hu}-$ man and bacterial orthologous gene clusters [21] using Ublast 
algorithm v. 7.0.1001 [22] with default parameters, except $e$-value $=0.000001$, accel (search speed at a cost for sensitivity) $=1$, maxaccepts (search termination) $=0$, and maxhits (maximum number of hits in the output) $=1$. Search results with an $e$-value below $10^{-30}$ were accepted. The data were processed with the MetaProteomeAnalyzer software (MPA; https://code.google.com/p/meta-proteome-analyzer/) [23] and further managed and analyzed with $R$ v. 3.1.1 (R-core team, $R$ : a language and environment for statistical computing. $R$ foundation for statistical computing, Vienna, Austria; available at http://www.R-project.org/.) [24] using in-house scripts.

Peptides were taxonomically assigned according to the lowest common ancestor (LCA) principle by using the online tool Unipept 2.3 [25] (settings: $I$ and $L$ equated; advanced missed cleavage option; and duplicates filtered). Relative percentages of bacterial phyla were calculated by summing the number of spectra per phylum divided by the overall number of spectra to which a bacterial phylum could be assigned.

\subsection{Phylogenetic microarray analysis}

The microbial composition of the faecal samples was assessed by a phylogenetic microarray (human intestinal tract chip) as described previously [14]. The raw data files were used and processed as described recently [26].

\subsection{Statistical analysis}

Principal coordinate analysis (PCoA) was run on NOGs count data with $R$ v. 3.1.1 using function PCoA in package ape [27]. The count data were filtered to remove NOGs with less than seven counts in any of the samples and $\log 10$ transformed. Similarity of samples was analyzed by calculating correlations that were transformed into distances as 1-r (Pearson's correlation coefficient). The PCoA sampling distributions for means of first and second vectors of PCoA of obese and nonobese were obtained by leave-one-out jackknife with 1000 iterations, where the PCoA was recomputed in each iteration step. The jackknife sampling distributions of means were analyzed with the one-way Wilcoxon rank-sum test to test for statistical significance between obese and nonobese separation in PCoA. To identify the separating NOGs and genus-like taxa in the PCoAs, a redundancy analysis using bootstrap aggregation (bagged RDA) was applied, as described recently [28] ( $p$-values $<0.05)$.

Correlation analysis was performed using the $R$-function cor.test. When a measurement value was missing for a sample, this sample was excluded from the analysis. A Benjamini-Hochberg adjusted correlation $p$-value lesser than 0.05 was chosen as a criterion for statistically significant differences, and only these results were reported.

Other between-group tests were performed with the oneway Wilcoxon rank-sum test, and differences were considered significant when the $p$-value was lesser than 0.05 .

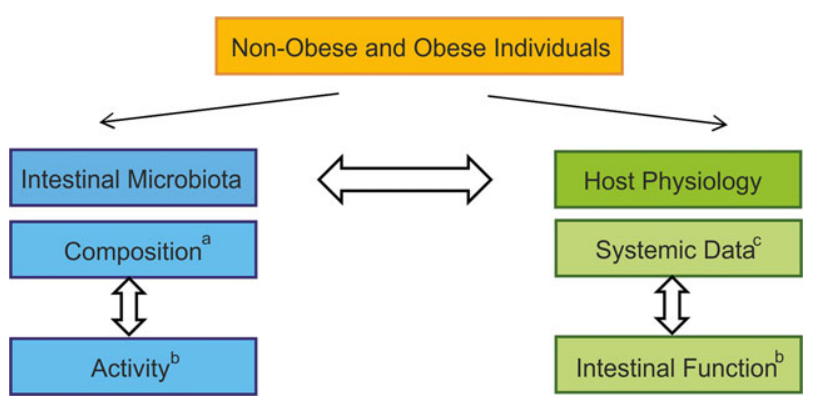

Figure 1. Study overview of 13 nonobese $(\mathrm{BMI}$ median = $22.8 \mathrm{~kg} / \mathrm{m}^{2}$ ) and 16 obese (BMI median $=42.3 \mathrm{~kg} / \mathrm{m}^{2}$ ) individuals. The composition and activity of the intestinal microbiota as well as host physiology were studied (a: phylogenetic analysis [14]; b: metaproteomics assessing microbial and human proteins (in this study); and c: blood measurements [14]).

\section{Results and discussion}

Due to its large impact on the health care system and society in general, there is an increasing need to address all possible factors that contribute to obesity. The human intestinal tract is one of the key organs to study. The colonic metaproteomics approach described here has the advantage of simultaneously focusing on two possible key players in the digestive system: the human host and the intestinal microbiota. We embarked on analyzing faecal samples from a cohort, of which health indicators and microbiota composition had been assessed previously [14]. We clustered subjects into a nonobese group $\left(\mathrm{BMI}<30 \mathrm{~kg} / \mathrm{m}^{2} ;\right.$ median $\left.=22.8 \mathrm{~kg} / \mathrm{m}^{2}\right)$ and an obese group with the majority having a BMI larger than $40 \mathrm{~kg} / \mathrm{m}^{2}$ (BMI $>30 \mathrm{~kg} / \mathrm{m}^{2} ;$ median $=42.3 \mathrm{~kg} / \mathrm{m}^{2}$ ). Subsequently, we collected all individual microbial and host metaproteomics data and generated a comprehensive dataset that allowed multiple comparisons (Fig. 1). In order to get an integrated insight into what differentiates obese from nonobese individuals, datasets were correlated.

\subsection{Combination of two search engines increased the MS/MS identification ratio and EggNOG annotation provided a high annotation rate}

Mass spectrometric analysis of proteins derived from faecal samples from 29 lean and obese adults generated a total of 893007 MS/MS spectra (Supporting Information Table S3). By applying two search engines, on average $27.39 \%$ (range: 16.49-32.68\%) of the MS/MS spectra were identified. Out of these, $91.86 \%$ (range: $87.64-96.03 \%$ ) could be mapped to an NOG at an $e$-value lower than $10^{-30}$ and $73.90 \%$ (range: $65.01-81.36 \%$ ) could be assigned to phylum level or lower (Supporting Information Tables S4 and S5). The combination of the result of two different search engines improved the identification ratio compared to earlier metaproteomic studies [29]. 

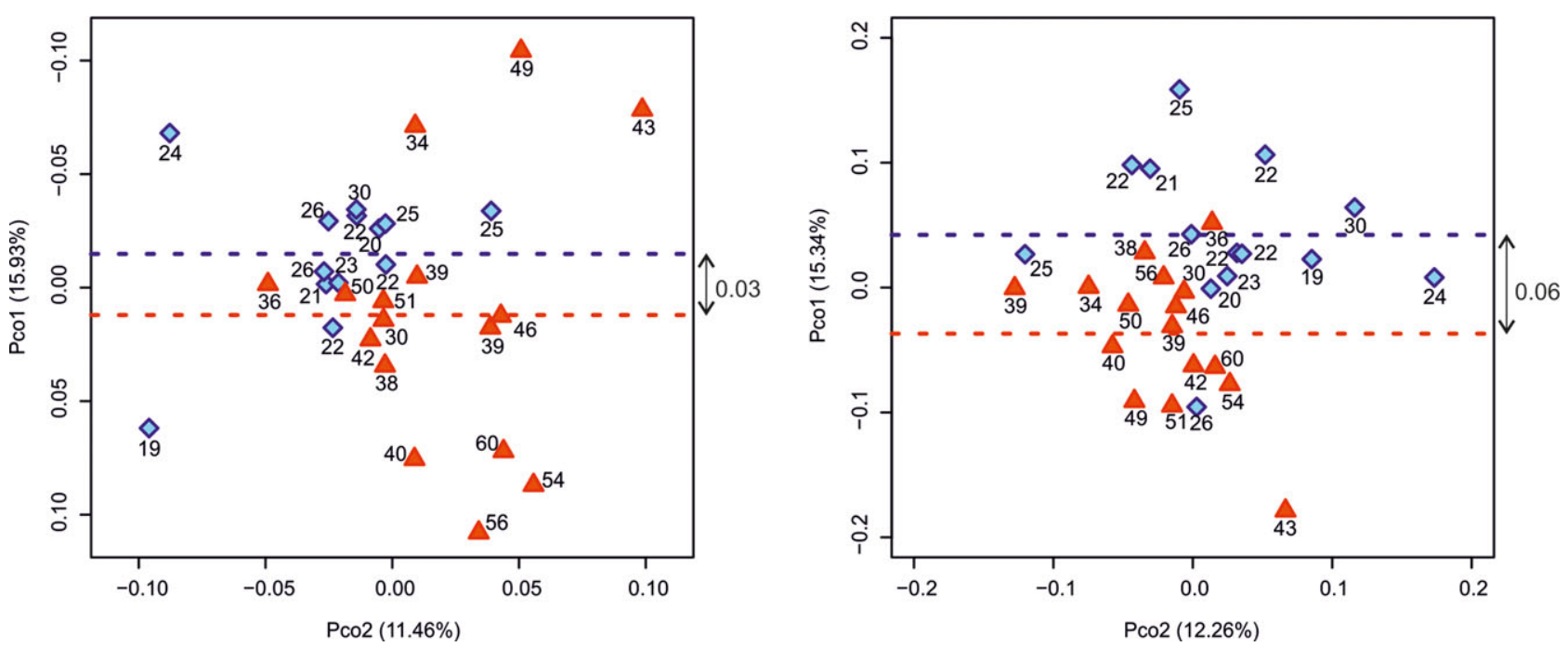

Figure 2. PCoA of bacterial composition data (based on phylogenetic microarray genus-like data; left panel) and bacterial NOGs (right panel) of faecal samples from nonobese (blue) and obese subjects (red). The actual BMI, in integers, of each subject is indicated. A significant difference in PCo1 was observed between obese and nonobese groups by using a jackknife approach ( $p$-values 0.001253 and $7.5 e-05$ for the composition and metaproteome datasets, respectively). The lines denote the means of the groups on the PCo1 and the arrows indicate the differences between these means.

\subsection{Bacterial NOGs separate nonobese and obese individuals}

The colonic metaproteome of nonobese subjects differed significantly from that of the obese subjects based on unsupervised ordination; PCoA was used to visualize variation among the samples (Fig. 2). In order to identify the components responsible for the separation, we applied bagged RDA, a supervised method, which identified 25 bacterial NOGs that significantly differentiated the nonobese and obese metaproteomes (Supporting Information Table S6). One NOG represented lipoproteins and another NOG proteins involved in sulfur-metabolism (NOG "cystathionine"), which were either higher or lower in the obese subjects as compared to the nonobese controls, respectively. However, the majority of NOGs related to carbohydrate metabolism where marked differences between obese and healthy subjects were evident. Peptides derived from proteins involved in (complex) C5 and C6 carbohydrate metabolism, including enolase, ribulokinase, xylulokinase, phosphoketolase, and a specific glycoside hydrolase were more abundant in nonobese subjects, while the obese subjects had much more proteins involved in starch and pectin metabolism, as deduced from the higher levels of peptides derived from $\alpha$-glucosidase and pectate lyase. This suggests that obese subjects consumed more starch and pectin, pointing to dietary habits explaining the differences in microbial activity.

Similarly to the metaproteomics data we applied PCoA to the previously determined phylogenetic data. The genuslevel data showed a significant separation between obese and nonobese individuals, confirming the previous findings at oligonucleotide level [14] (Fig. 2). However, at the peptide level, the groups differed more pronouncedly, reflected by a twofold increased distance of the means of the first principle coordinate PCo1, indicating a stronger separating power of the functional data compared to the compositional data.

\subsection{Less but more active Bacteroidetes in obese compared to nonobese individuals}

Phylogenetic microarray analysis identified significantly lower levels of Bacteroidetes in the studied obese compared to nonobese individuals ( 11.65 versus $21.94 \%$ at a $p$-value < 0.05; Supporting Information Table 7). This finding was compared to the LCA analysis based on the metaproteome data (Supporting Information Table 7). By determining the spectral counts of Bacteroidetes peptides and computing from these the fraction compared to the other bacterial phyla, we observed a 1.2-fold higher mean in the obese than the nonobese subjects, which was, however, not significant (26.27 versus $21.61 \%$, Supporting Information Table 7). However, when computing the ratios of the relative percentages for Bacteroidetes based on the metaproteomics and phylogenetic analysis (Fig. 3), a significant difference between nonobese and obese subjects was identified, indicative of a higher activity of Bacteroidetes in the obese compared to the nonobese group. One would expect that such high activity would lead to an outgrowth of the Bacteroidetes, but the phylogenetic data indicate that this is not the case. A simple explanation might relate to the growth rate of Bacteroides spp., which was found to be highly affected by the environmental $\mathrm{pH}$ [30]. The level of SCFA has been reported to be significantly higher in obese as compared to nonobese subjects [31], and this is likely to 


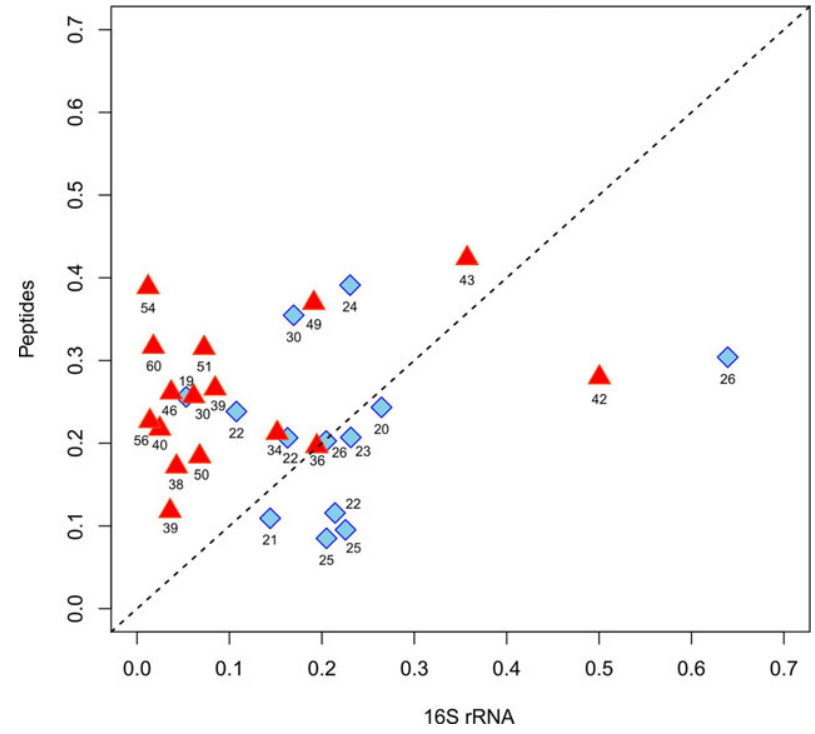

Figure 3. Relative amount of Bacteroidetes as determined by phylogenetic microarray analysis ( $x$-axis) and metaproteome analysis ( $y$-axis) in nonobese (blue) and obese individuals (red); the actual BMI (in integers) of each subject is indicated. The difference between the groups was found to be significant ( $p$-value 0.0037 in the one-way Wilcoxon rank-sum test).

lower the colonic $\mathrm{pH}$ and hence affect the growth of Bacteroides spp. that hardly grow below pH 5.5 [30].

Since the conclusion on the biological activity of Bacteroidetes is based on the combination of metaproteome and phylogenetic data, one needs to consider potential confounding factors that may contribute to the differences. A technical bias due to nonuniform lysis and subsequent DNA degradation has been suggested to occur with Bacteroides spp. during long-term freezer storage [32]. However, this bias is unlikely to differ between the faecal samples of lean and obese individuals and to affect protein stability, hence the observed increased abundance of Bacteroidetes proteins in the obese subjects is likely to be genuine. In addition, we used nonseparated faecal material for protein extraction and this obviates one bias that recently has been reported, as it was shown that Bacteroidaceae was underrepresented in a metaproteomics study when bacterial pellets were targeted, probably because of the binding of Bacteroidetes to food particles [33]. In conclusion, Bacteroidetes peptides can be identified in high numbers (up to $40 \%$ of the spectra with bacterial phylum-specific assignment) in faecal samples of the obese individuals. Thus, Bacteroidetes appear to be very active in obese individuals. Host factors, a diet favoring Bacteroidetes activity, as well as a specific microbial composition in obese individuals could be responsible for this finding, while the outgrowth of the Bacteroidetes is likely to be limited by a low $\mathrm{pH}$.

Within Bacteroidetes, the two genera Bacteroides and Prevotella are of specific interest due to differences in their substrate preferences as well as metabolic output. Hence, we addressed the activity of these two genera. Overall, the relative distribution of Bacteroides and Prevotella corresponded well between the phylogenetic and metaproteome data, as there was no significant difference in the Bacteroides/Prevotella ratio between the nonobese and obese group, indicative of a constant activity of bacteria belonging to these groups (Supporting Information Fig. 1). In faecal samples of several obese individuals, a high number of peptides derived from Prevotella were detected. However, there was a trend of less active Prevotella in the individuals with extreme BMI (>47).

Supervised identification, using bagged RDA, of differences between the nonobese and obese group revealed a significant difference in the Bacteroidetes NOG aminoacylhistidine dipeptidase (Supporting Information Table 6). High levels of peptides derived from this enzyme were found in the obese group. Bacterial proteinases have been suspected to be involved in inflammatory bowel disease (IBD) [34]. Hence, it is of interest to note that the very same obese subjects studied here show an increase of serum C-reactive protein, a well-established marker of systemic inflammation.

Since the obese and nonobese groups differed clearly based on their metaproteome and phylogenetic composition (Figs. 2 and 3), we also addressed the common features of both groups by defining the core metaproteome based on the bacterial NOGs (Supporting Information Fig. 2). The core metaproteomes of the obese and nonobese groups were highly similar and included the same major group (63\% in the obese group and $65 \%$ in the nonobese group) consisting of proteins predicted to be involved in energy production and conversion, amino acid transport metabolism, and inorganic ion transport and metabolism (cluster of orthologous genes (COG) families G, E, P, and R). The extracellular solute-binding protein family 1 and a solute-binding protein were found next to the often reported glutamate dehydrogenase [11] as the top three most abundant representatives. This reflects the high transport activity of the intestinal bacteria. Among the other abundant proteins, a few NOG representatives stood out, although their abundance was highly similar. These included proteins related to the Ragb Susd domain-containing proteins and the susB protein that are all abundant in Bacteroides spp. and related to sugar binding and transport. In conclusion, no significant differences in the microbial core functions between the obese and nonobese group were observed, most likely reflecting the fact that these core functions are housekeeping functions that are present in all intestinal bacteria.

\subsection{Human NOGs}

Clustering the samples according to their counts per human NOGs also separated obese and nonobese samples in an unsupervised PCoA with only few exceptions (Fig. 4). Bagged RDA identified 41 human NOGs to significantly differentiate between obese and nonobese samples (Supporting Information Table 6). Several proteins were very abundant in faecal samples from obese subjects, including trehalase, alkaline phosphatase (AP), several serpins (serine protease inhibitors), 


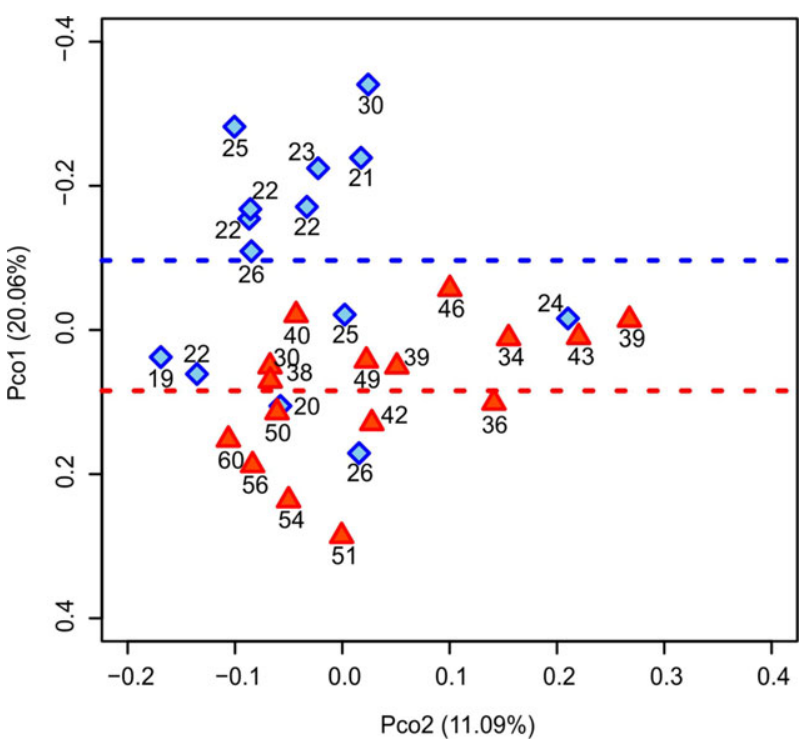

Figure 4. PCoA of human NOGs of faecal samples from nonobese (blue) and obese subjects (red). The actual BMI (in integers) of each subject is indicated. A significant difference in PCo1 was observed between obese and nonobese groups by using a jackknife approach ( $p$-value 0.00054).

and $\alpha$-amylase. Trehalose is a disaccharide, which acts as a compatible solute and is formed in bacteria upon stress to dehydration, such as a lower osmotic value that may be produced from a high-fat diet as known from mice studies [35]. The thus formed trehalose could trigger upregulation of trehalase, which is a brush border enzyme degrading trehalose. Alternatively, as trehalase is a marker for enterocyte development, its abundance in obese subjects could be explained by increased intestinal injury [36,37], consistent with the increase in intestinal damage markers described in severely obese hyperglycemic subjects [38] and with the intestinal inflammation described in the subjects studied here [14].

Intestinal AP has been linked in various ways with the intestinal microbiota and is generally assumed to detoxify lipopolysaccharides [39]. Hence, the abundance of AP in obese subjects may be indicative of high levels of lipopolysaccharides and the resulting endotoxemia, which is supported by elevated C-reactive protein levels in this group [14]. It may be part of an endogenous mechanism compensating for metabolic changes. In mice, AP was shown to protect against metabolic syndrome [40]. It would be of interest to also address the serum AP concentration in these subjects, since it has been reported that AP levels may be elevated in obesity [41]. Intestinal or serum AP could be a biomarker for a metabolic imbalance.

The higher levels of serpins in the obese subjects can be seen as an indicator of high levels of serine proteases that need to be complexed. It is known from other inflammatory diseases, such as IBD, that the level of serine proteases is highly increased [34]. Extremely high numbers (>1000) of identified spectra for $\alpha$-amylase were found in several of the obese individuals. This points to a high capacity of the intestinal system to digest starch and suggests a high amount of starch in the diet of obese individuals, which is corroborated by the high level of $\alpha$-glucosidase in the bacterial metaproteome (as stated in Section 3.2).

Altogether, the overrepresentation of several inflammation-linked proteins in the obese group compared to the nonobese can be seen as an indication of perturbation that is compatible with the idea of a compromised gut in obesity.

\subsection{Insulin sensitivity correlates with bacterial NOGs}

When correlating clinical parameters with either human or bacterial NOGs, no associations with BMI were identified (data not shown). However, insulin sensitivity (as indicated by homeostasis model assessment values) correlated significantly with some of the metaproteome data. The higher the homeostasis model assessment was, i.e. the more insulinresistant a person was, the higher were the levels of the NOG comprising two component regulators (correlation factor $0.757, p$-value $<0.05$ ) and pectate lyase (correlation factor $0.871, p$-value $<0.05)$. Two component regulators are canonical bacterial sensing systems, consisting of a membranelocated sensor kinase and an intracellular response regulator. Notably, the sensor kinases may interact with the host and it is of interest to note that the LCA analysis indicated that the majority of peptides mapping to this group of proteins derived from bacteria from the genus Ruminococcus. Several Ruminococcus spp. such as $R$. gnavus have been implicated in irritable bowel syndrome and mucosal inflammation in IBD [42].

Pectate lyase is a key enzyme involved in pectin degradation and LCA analysis implied bacteria belonging to the genera Eubacterium or Bacteroides as its source. It remains to be determined whether this reflects simply an increase in pectin metabolism in obese subjects or points to a link between specific pectin degradation products and metabolic signaling involved in insulin resistance.

\section{Concluding remarks}

The role of the intestinal microbiota in obesity has been studied since the last decade [43]. Apart from a case report in adolescents [13], this is the first study that describes the intestinal metaproteome of nonobese and obese subjects and goes beyond phylogenetic and metagenomic analysis of the intestinal microbiota in obese adults. Earlier studies revealed differences in the intestinal microbial composition between lean and obese individuals. However, the finding that the overall microbial activity separates obese and nonobese individuals provides a new dimension to the much researched area of obesity. We also show that both microbial and human protein data, and the combination thereof, contributed 
to identifying differences in obese versus nonobese individuals even more than phylogenetic data (Figs. 2 and 4). We discovered a significant separation of nonobese and obese individuals based on a functional characterization of intestinal microbiota and the human intestinal proteome, exceeding the differences observed at the composition level (Fig. 3). Although the phylum Bacteroidetes was less abundant in the obese group, high numbers of Bacteroidetes peptides were identified implying high activity of this phylum that may have a restricted growth potential because of the low luminal $\mathrm{pH}$ in obese subjects. This finding is of significance because of the initial findings on the microbiota in obesity that suggested a lower abundance of Bacteroidetes in obese subjects [44]. Our data indicate that this is indeed the case, notably in morbidly obese subjects, but the metaproteome data suggest that the phylum Bacteroidetes may contribute substantially to the overall functional activities in the lower intestinal tract. The fact that BMI did not correlate with any of the NOGs indicates that other factors than BMI are involved. One driving factor of the microbiota and its activity is diet and this is likely to be important here as well, notably as it appeared that the colon of obese subjects is enriched for proteins involved in starch and pectin degradation. A confounding factor that we could not eliminate was the use of regular medication between the obese and the nonobese group, which may also have affected the microbiota in individual cases. However, grouping individuals in nonobese and obese groups may unify metabolic similarities allowing detecting differences between these two groups, as is shown here.

Longitudinal studies with a critical number of subjects carefully monitoring microbiota development and its actual functionality taking various environmental factors into account such as the colonic $\mathrm{pH}$ are required to answer the chicken-or-the-egg question: Is the microbiota causing a difference in metabolism that leads to an energetic misbalance, or are differences in metabolism and/or eating habits causing a change in microbiota? The current study shows that metaproteomics is an important addition to the toolbox that enables the simultaneous determination of microbial and host activity in the intestinal tract.

Runi Erbil, Outi Immonen, and Nahaison Krips are thanked for technical assistance, Mark de Been for sharing the HIMPdb, and Jarkko Salojärvi for statistical advice and sharing the bagged RDA script.

Funding was provided by the Doctoral Programme in Food Chain and Health, the Research Foundation of the University of Helsinki, the Finnish Academy of Science (grants 141130, 137389, and 141140), the European Research Council (grant 250172-MicrobesInside), the Dutch Digestive Foundation (project grant WO 09-46) the European Commission 7th Framework Programme (grant 262067-PRIME-XS), and Ghent University (Multidisciplinary Research Partnership "Bioinformatics: from nucleotides to networks").

The authors have declared no conflict of interest.

\section{References}

[1] Stenvinkel, P., Obesity - a disease with many aetiologies disguised in the same oversized phenotype: has the overeating theory failed? Nephrol. Dial. Transplant. 2014, ePub ahead of print, doi: 10.1093/ndt/gfu338.

[2] Finucane, M. M., Sharpton, T. J., Laurent, T. J., Pollard, K. S., A taxonomic signature of obesity in the microbiome? Getting to the guts of the matter. PLoS One 2014, 9, e84689.

[3] Moran, C. P., Shanahan, F., Gut microbiota and obesity: role in aetiology and potential therapeutic target. Best Pract. Res. Clin. Gastroenterol. 2014, 28, 585-597.

[4] Tremaroli, V., Bäckhed, F., Functional interactions between the gut microbiota and host metabolism. Nature 2012, 489 242-249.

[5] Tims, S., Derom, C., Jonkers, D. M., Vlietinck, R. et al., Microbiota conservation and $\mathrm{BMI}$ signatures in adult monozygotic twins. ISME J. 2012, 7, 707-717.

[6] Walters, W. A., Xu, Z., Knight, R., Meta-analyses of human gut microbes associated with obesity and IBD. FEBS Lett. 2014, 588, 4223-4233.

[7] Zoetendal, E. G., Raes, J., van den Bogert, B., Arumugam, M. et al., The human small intestinal microbiota is driven by rapid uptake and conversion of simple carbohydrates. ISME J. 2012, 6, 1415-1426.

[8] Franzosa, E. A., Morgan, X. C., Segata, N., Waldron, L. et al., Relating the metatranscriptome and metagenome of the human gut. Proc. Natl. Acad. Sci. USA 2014, 111, E2329-E2338.

[9] Verberkmoes, N. C., Russell, A. L., Shah, M., Godzik, A. et al., Shotgun metaproteomics of the human distal gut microbiota. ISME J. 2009, 3, 179-189.

[10] Rooijers, K., Kolmeder, C., Juste, C., Doré, J. et al., An iterative workflow for mining the human intestinal metaproteome. BMC Genomics 2011, 12, 1-11.

[11] Kolmeder, C. A., de Been, M., Nikkilä, J., Ritamo, I. et al., Comparative metaproteomics and diversity analysis of human intestinal microbiota testifies for its temporal stability and expression of core functions. PLoS One 2012, 7, e29913.

[12] Pérez-Cobas, A. E., Gosalbes, M. J., Friedrichs, A., Knecht, H. et al., Gut microbiota disturbance during antibiotic therapy: a multiomic approach. Gut 2013, 62, 1591-1601.

[13] Ferrer, M., Ruiz, A., Lanza, F., Haange, S. et al., Microbiota from the distal guts of lean and obese adolescents exhibit partial functional redundancy besides clear differences in community structure. Environ. Microbiol. 2013, 15, 211-226.

[14] Verdam, F. J., Fuentes, S., de Jonge, C., Zoetendal, E. G. et al., Human intestinal microbiota composition is associated with local and systemic inflammation in obesity. Obesity 2013, 21, E607-E615.

[15] Muth, T., Kolmeder, C. A., Salojärvi, J., Keskitalo, S. et al., Navigating through metaproteomics data - a logbook of database searching. Proteomics 2015, 15, 3439-3453.

[16] Shevchenko, A., Tomas, H., Havlis, J., Olsen, J. V. et al., In-gel digestion for mass spectrometric characterization of proteins and proteomes. Nat. Protoc. 2006, 1, 2856-2860. 
[17] Craig, R., Beavis, R. C., Tandem: matching proteins with tandem mass spectra. Bioinformatics 2004, 20, 1466-1467.

[18] Geer, L. Y., Markey, S. P., Kowalak, J. A., Wagner, L. et al., Open mass spectrometry search algorithm. J. Proteome Res. 2004, 3, 958-964.

[19] Käll, L., Storey, J. D., MacCoss, M. J., Noble, W. S., Posterior error probabilities and false discovery rates: two sides of the same coin. J. Proteome Res. 2007, 7, 40-44.

[20] Ng, C., DeMaere, M. Z., Williams, T. J., Lauro, F. M. et al., Metaproteogenomic analysis of a dominant green sulfur bacterium from Ace Lake. ISME J. 2010, 4, 1002-1019.

[21] Powell, S., Forslund, K., Szklarczyk, D., Trachana, K. et al., EggNOG v4.0: nested orthology inference across 3686 organisms. Nucleic Acids Res. 2014, 42, D231-D239.

[22] Edgar, R. C., Search and clustering orders of magnitude faster than BLAST. Bioinformatics 2010, 26, 2460-2461.

[23] Muth, T., Behne, A., Heyer, R., Kohrs, F. et al., The MetaProteomeAnalyzer: a powerful open-source software suite for metaproteomics data analysis and interpretation. J. Proteome Res. 2015, 14, 1557-1565.

[24] Pages, H., Aboyoun, P., Gentleman, R., DebRoy, S., Biostrings: string objects representing biological sequences, and matching algorithms. $R$ package version 2014, 2.32.1.

[25] Mesuere, B., Devreese, B., Debyser, G., Aerts, M. et al., Unipept: tryptic peptide-based biodiversity analysis of metaproteome samples. J. Proteome Res. 2012, 11, 57735780.

[26] Lahti, L., Torrente, A., Elo, L. L., Brazma, A. et al., A fully scalable online pre-processing algorithm for short oligonucleotide microarray atlases. Nucleic Acids Res. 2013, 41, 110.

[27] Paradis, E., Claude, J., Strimmer, K., APE: analyses of phylogenetics and evolution in $\mathrm{R}$ language. Bioinformatics 2004, 20, 289-290.

[28] Jalanka-Tuovinen, J., Salojärvi, J., Salonen, A., Immonen, O. et al., Faecal microbiota composition and host-microbe cross-talk following gastroenteritis and in postinfectious irritable bowel syndrome. Gut 2014, 63, 1737-1745.

[29] Cantarel, B. L., Erickson, A. R., Verberkmoes, N. C., Erickson, B. K. et al., Strategies for metagenomic-guided whole-community proteomics of complex microbial environments. PLoS One 2011, 6, e27173.

[30] Duncan, S. H., Louis, P., Thomson, J. M., Flint, H. J., The role of $\mathrm{pH}$ in determining the species composition of the human colonic microbiota. Environ. Microbiol. 2009, 11, 2112-2122.
[31] Fernandes, J., Su, W., Rahat-Rozenbloom, S., Wolever, T. et al., Adiposity, gut microbiota and faecal short chain fatty acids are linked in adult humans. Nutr. Diabetes 2014, 4, e121.

[32] Bahl, M. I., Bergström, A., Licht, T. R., Freezing fecal samples prior to DNA extraction affects the Firmicutes to Bacteroidetes ratio determined by downstream quantitative PCR analysis. FEMS Microbiol. Lett. 2012, 329, 193-197.

[33] Tanca, A., Palomba, A., Pisanu, S., Addis, M. F. et al., Enrichment or depletion? The impact of stool pretreatment on metaproteomic characterization of the human gut microbiota. Proteomics 2015, 15, 3473-3485.

[34] Steck, N., Mueller, K., Schemann, M., Haller, D., Bacterial proteases in IBD and IBS. Gut 2012, 61, 1610-1618.

[35] Daniel, H., Gholami, A. M., Berry, D., Desmarchelier, C. et al., High-fat diet alters gut microbiota physiology in mice. ISME J. 2014, 8, 295-308.

[36] Nanthakumar, N. N., Dai, D., Meng, D., Chaudry, N. et al., Regulation of intestinal ontogeny: effect of glucocorticoids and luminal microbes on galactosyltransferase and trehalase induction in mice. Glycobiology 2005, 15, 221-232.

[37] Tóth Jr, Š, Pekárová, T., Varga, J., Tomečková, V. et al., Trehalase as a possible marker of intestinal ischemia-reperfusion injury. Acta Biochim. Pol. 2013, 60, 411-416.

[38] Verdam, F. J., Greve, J. W. M., Roosta, S., van Eijk, H. et al., Small intestinal alterations in severely obese hyperglycemic subjects. J. Clin. Endocrinol. Metab. 2010, 96, E379-E383.

[39] Lallès, J., Intestinal alkaline phosphatase: novel functions and protective effects. Nutr. Rev. 2014, 72, 82-94.

[40] Kaliannan, K., Hamarneh, S. R., Economopoulos, K. P., Nasrin Alam, S. et al., Intestinal alkaline phosphatase prevents metabolic syndrome in mice. Proc. Natl. Acad. Sci. USA 2013, 110, 7003-7008.

[41] Golik, A., Rubio, A., Weintraub, M., Byrne, L., Elevated serum liver enzymes in obesity: a dilemma during clinical trials. Int. J. Obes. 1991, 15, 797-801.

[42] Png, C. W., Lindén, S. K., Gilshenan, K. S., Zoetendal, E. G. et al., Mucolytic bacteria with increased prevalence in IBD mucosa augment in vitro utilization of mucin by other bacteria. Am. J. Gastroenterol. 2010, 105, 2420-2428.

[43] Turnbaugh, P. J., Ley, R. E., Mahowald, M. A., Magrini, V. et al., An obesity-associated gut microbiome with increased capacity for energy harvest. Nature 2006, 444, 1027-1031.

[44] Ley, R. E., Bäckhed, F., Turnbaugh, P., Lozupone, C. A. et al., Obesity alters gut microbial ecology. Proc. Natl. Acad. Sci. USA 2005, 102, 11070-11075. 